Int. J. Number Theory 12(2016), no. 2, 527-539.

\title{
TWO CONGRUENCES INVOLVING HARMONIC NUMBERS WITH APPLICATIONS
}

\author{
GUO-SHUAI MAO AND ZHI-WEI SUN
}

\begin{abstract}
The harmonic numbers $H_{n}=\sum_{0<k \leqslant n} 1 / k(n=0,1,2, \ldots)$ play important roles in mathematics. Let $p>3$ be a prime. With helps of some combinatorial identities, we establish the following two new congruences:

$$
\sum_{k=1}^{p-1} \frac{\left(\begin{array}{c}
2 k \\
k
\end{array}\right)}{k} H_{k} \equiv \frac{1}{3}\left(\frac{p}{3}\right) B_{p-2}\left(\frac{1}{3}\right)(\bmod p)
$$
\end{abstract}

and

$$
\sum_{k=1}^{p-1} \frac{\left(\begin{array}{c}
2 k \\
k
\end{array}\right)}{k} H_{2 k} \equiv \frac{7}{12}\left(\frac{p}{3}\right) B_{p-2}\left(\frac{1}{3}\right)(\bmod p),
$$

where $B_{n}(x)$ denotes the Bernoulli polynomial of degree $n$. As an application, we determine $\sum_{n=1}^{p-1} g_{n}$ and $\sum_{n=1}^{p-1} h_{n}$ modulo $p^{3}$, where

$$
g_{n}=\sum_{k=0}^{n}\left(\begin{array}{l}
n \\
k
\end{array}\right)^{2}\left(\begin{array}{c}
2 k \\
k
\end{array}\right) \quad \text { and } \quad h_{n}=\sum_{k=0}^{n}\left(\begin{array}{l}
n \\
k
\end{array}\right)^{2} C_{k}
$$

with $C_{k}=\left(\begin{array}{c}2 k \\ k\end{array}\right) /(k+1)$.

\section{INTRODUCTION}

For $n \in \mathbb{N}=\{0,1,2, \ldots\}$, define

$$
H_{n}:=\sum_{0<k \leqslant n} \frac{1}{k} \text { and } H_{n}^{(2)}:=\sum_{0<k \leqslant n} \frac{1}{k^{2}} .
$$

Those $H_{n}$ with $n \in \mathbb{N}$ are the classical harmonic numbers, and those $H_{n}^{(2)}$ with $n \in \mathbb{N}$ are called the second-order harmonic numbers.

Let $p>3$ be a prime. By a classical result of J. Wolstenholme [W], we have

$$
H_{p-1} \equiv 0\left(\bmod p^{2}\right) \text { and } H_{p-1}^{(2)} \equiv 0(\bmod p),
$$

Key words and phrases. Central binomial coefficients, congruences, harmonic numbers, Bernoulli polynomials.

2010 Mathematics Subject Classification. Primary 11B65, 11B68; Secondary 05A10, $11 \mathrm{~A} 07$.

The second author is the corresponding author. This research was supported by the Natural Science Foundation of China (grant 11571162). 
which imply that

$$
\frac{1}{2}\left(\begin{array}{c}
2 p \\
p
\end{array}\right)=\left(\begin{array}{c}
2 p-1 \\
p-1
\end{array}\right) \equiv 1\left(\bmod p^{3}\right) .
$$

Z.-W. Sun [S12a] established some fundamental congruences involving harmonic numbers; for example, he showed that $\sum_{k=1}^{p-1} H_{k} /\left(k 2^{k}\right) \equiv$ $0(\bmod p)$ motivated by the known identity $\sum_{k=1}^{\infty} H_{k} /\left(k 2^{k}\right)=\pi^{2} / 12$.

Let $p>3$ be a prime. By Sun and R. Tauraso [ST11, (1.9)], and Sun [S12c, (2.9)], we have

$$
\sum_{k=0}^{p-1}\left(\begin{array}{c}
2 k \\
k
\end{array}\right) \equiv\left(\frac{p}{3}\right)\left(\bmod p^{2}\right)
$$

and

$$
\sum_{k=0}^{p-1}(-1)^{k}\left(\begin{array}{c}
p-1 \\
k
\end{array}\right)\left(\begin{array}{c}
2 k \\
k
\end{array}\right) \equiv 3^{p-1}\left(\frac{p}{3}\right)\left(\bmod p^{2}\right)
$$

respectively, where $(-)$ denotes the Legendre symbol. Hence

$$
\sum_{k=0}^{p-1}\left(\begin{array}{c}
2 k \\
k
\end{array}\right) H_{k} \equiv\left(\frac{p}{3}\right) \frac{1-3^{p-1}}{p}(\bmod p)
$$

since

$$
(-1)^{k}\left(\begin{array}{c}
p-1 \\
k
\end{array}\right)=\prod_{0<j \leqslant k}\left(1-\frac{p}{j}\right) \equiv 1-p H_{k}\left(\bmod p^{2}\right)
$$

for all $k=0,1, \ldots, p-1$. In 2010 Sun and Tauraso [ST10] proved that

$$
\sum_{k=1}^{p-1} \frac{\left(\begin{array}{c}
2 k \\
k
\end{array}\right)}{k} \equiv \frac{8}{9} p^{2} B_{p-3}\left(\bmod p^{3}\right)
$$

where $B_{0}, B_{1}, B_{2}, \ldots$ are the Bernoulli numbers given by

$$
\frac{x}{e^{x}-1}=\sum_{n=0}^{\infty} B_{n} \frac{x^{n}}{n !} \quad(0<|x|<2 \pi) .
$$

In 2011 Sun [S11] showed that

$$
\sum_{k=1}^{(p-1) / 2} \frac{\left(\begin{array}{c}
2 k \\
k
\end{array}\right)}{k} \equiv-\left(\frac{-1}{p}\right) \frac{8}{3} p E_{p-3}\left(\bmod p^{2}\right),
$$

where $(\dot{\bar{p}})$ denotes the Legendre symbol and $E_{p-3}$ stands for the $(p-3)$ th Euler number. 
Recall that the Bernoulli polynomials are given by

$$
B_{n}(x)=\sum_{k=0}^{n}\left(\begin{array}{l}
n \\
k
\end{array}\right) B_{k} x^{n-k} \quad(n=0,1,2, \ldots) .
$$

Motivated by the above work, we mainly obtain the following result in this paper.

Theorem 1.1. Let $p>3$ be a prime. Then

$$
\sum_{k=1}^{p-1} \frac{\left(\begin{array}{c}
2 k \\
k
\end{array}\right)}{k} H_{k} \equiv \frac{1}{3}\left(\frac{p}{3}\right) B_{p-2}\left(\frac{1}{3}\right)(\bmod p)
$$

and

$$
\sum_{k=1}^{p-1} \frac{\left(\begin{array}{c}
2 k \\
k
\end{array}\right)}{k} H_{2 k} \equiv \frac{7}{12}\left(\frac{p}{3}\right) B_{p-2}\left(\frac{1}{3}\right)(\bmod p) .
$$

Remark 1.1. Another motivation of Theorem 1.1 is our desire to prove Theorem 1.2 in this section. Why (1.1) and (1.2) should involve the value of $B_{p-2}(x)$ at $1 / 3$ ? There are no intuitive reasons, but a slight indication comes from the following congruence in $[\mathrm{ST} 11,(1.5)]$ for any prime $p>3$ :

$$
\sum_{k=0}^{p-1}\left(\begin{array}{c}
2 k \\
k
\end{array}\right) \equiv\left(\frac{p}{3}\right)+2 p \sum_{k=1}^{p-1} \frac{(-1)^{k}}{k}\left(\frac{p-k}{3}\right)\left(\bmod p^{3}\right) .
$$

There are no closed forms for the sums in (1.1) and (1.2). Our approach to Theorem 1.1 is somewhat unique in the sense that it depends heavily on some special combinatorial identities.

Clearly Theorem 1.1 has the following consequence.

Corollary 1.1. For any prime $p>3$ we have

$$
\sum_{k=1}^{p-1} \frac{\left(\begin{array}{c}
2 k \\
k
\end{array}\right)}{k}\left(4 H_{2 k}-7 H_{k}\right) \equiv 0(\bmod p)
$$

Recall that $H_{p-1} \equiv 0\left(\bmod p^{2}\right)$ for any prime $p>3$. So our following conjecture is much stronger than Corollary 1.1.

Conjecture 1.1. For any prime $p>3$ we have

$$
\sum_{k=1}^{p-1} \frac{\left(\begin{array}{c}
2 k \\
k
\end{array}\right)}{k}\left(4 H_{2 k}-7 H_{k}\right) \equiv-14 \frac{H_{p-1}}{p}+\frac{278}{15} p^{3} B_{p-5}\left(\bmod p^{4}\right) .
$$


Note that Theorem 1.1 and Corollary 1.1 are related to the second author's following conjectural formulas for $\zeta(3)=\sum_{n=1}^{\infty} 1 / n^{3}$ and $K=$ $\sum_{k=1}^{\infty}\left(\frac{k}{3}\right) / k^{2}(\mathrm{cf} .[\mathrm{S} 14 \mathrm{a}])$ :

$$
\sum_{k=1}^{\infty} \frac{H_{2 k}+2 H_{k}}{k^{2}\left(\begin{array}{c}
2 k \\
k
\end{array}\right)}=\frac{5}{3} \zeta(3) \text { and } \sum_{k=1}^{\infty} \frac{H_{2 k}+17 H_{k}}{k^{2}\left(\begin{array}{c}
2 k \\
k
\end{array}\right)}=\frac{5}{2} \sqrt{3} \pi K .
$$

The Franel numbers $f_{n}=\sum_{k=0}^{n}\left(\begin{array}{l}n \\ k\end{array}\right)^{3}(n=0,1,2, \ldots)$ play important roles in combinatorics and number theory. In 1975 P. Barrucand [B] obtained the identity $\sum_{k=0}^{n}\left(\begin{array}{l}n \\ k\end{array}\right) f_{k}=g_{n}$, where

$$
g_{n}:=\sum_{k=0}^{n}\left(\begin{array}{l}
n \\
k
\end{array}\right)^{2}\left(\begin{array}{c}
2 k \\
k
\end{array}\right) .
$$

The sequences $\left(f_{n}\right)_{n \geqslant 0}$ and $\left(g_{n}\right)_{n \geqslant 0}$ are two of the five sporadic sequences (cf. D. Zagier [Z, Section 4]) which are integral solutions of certain Apéry-like recurrence equations and closely related to the theory of modular forms. In 2013, Sun [S13] revealed some unexpected connections between those numbers $f_{n}, g_{n}$ and representations of primes $p \equiv 1(\bmod 3)$ in the form $x^{2}+3 y^{2}$ with $x, y \in \mathbb{Z}$. For any prime $p>3$, Sun $[\mathrm{S} 14 \mathrm{~b}]$ and $[\mathrm{S} 12 \mathrm{~b},(1.15)]$ showed that

$$
\sum_{n=1}^{p-1} g_{n} \equiv \sum_{n=1}^{p-1} h_{n} \equiv 0\left(\bmod p^{2}\right)
$$

where

$$
h_{n}:=\sum_{k=0}^{n}\left(\begin{array}{l}
n \\
k
\end{array}\right)^{2} C_{k}
$$

and $C_{k}$ refers to the Catalan number $\left(\begin{array}{c}2 k \\ k\end{array}\right) /(k+1)=\left(\begin{array}{c}2 k \\ k\end{array}\right)-\left(\begin{array}{c}2 k \\ k+1\end{array}\right)$. The numbers $h_{0}, h_{1}, h_{2}, \ldots$ appeared naturally in the second author's study of Apéry polynomials (cf. [S12b]).

Applying Theorem 1.1, we deduce the following result.

Theorem 1.2. Let $p>3$ be a prime. Then

$$
\frac{1}{p^{2}} \sum_{k=1}^{p-1} g_{k} \equiv \sum_{k=1}^{p-1} g_{k} H_{k}^{(2)} \equiv \frac{5}{8}\left(\frac{p}{3}\right) B_{p-2}\left(\frac{1}{3}\right)(\bmod p)
$$

and

$$
\frac{1}{p^{2}} \sum_{k=1}^{p-1} h_{k} \equiv \sum_{k=1}^{p-1} h_{k} H_{k}^{(2)} \equiv \frac{3}{4}\left(\frac{p}{3}\right) B_{p-2}\left(\frac{1}{3}\right)(\bmod p) .
$$


We are going to prove Theorems 1.1 and 1.2 in Sections 2 and 3 respectively. Our proofs make use of some sophisticated combinatorial identities.

\section{Proof of Theorem 1.1}

Lemma 2.1. For any $n \in \mathbb{N}$, we have

$$
\begin{aligned}
\sum_{k=0}^{n}\left(\begin{array}{l}
x \\
k
\end{array}\right)\left(\begin{array}{c}
y \\
n-k
\end{array}\right) & =\left(\begin{array}{c}
x+y \\
n
\end{array}\right), \\
\sum_{k=0}^{n}\left(\begin{array}{l}
n \\
k
\end{array}\right)^{2} H_{k} & =\left(\begin{array}{c}
2 n \\
n
\end{array}\right)\left(2 H_{n}-H_{2 n}\right), \\
\sum_{k=0}^{n}(-1)^{k}\left(\begin{array}{l}
n \\
k
\end{array}\right)\left(\begin{array}{c}
2 k \\
k
\end{array}\right) & =(-1)^{n} \sum_{k=0}^{\lfloor n / 2\rfloor}\left(\begin{array}{c}
n \\
2 k
\end{array}\right)\left(\begin{array}{c}
2 k \\
k
\end{array}\right) .
\end{aligned}
$$

The three identities (2.1)-(2.3) are known, see, e.g., [G, (3.1), (3.125) and (3.86)].

Proof of Theorem 1.1. By (2.2),

$$
\sum_{j=1}^{k}\left(\begin{array}{c}
k \\
j
\end{array}\right)^{2} H_{j}=\left(\begin{array}{c}
2 k \\
k
\end{array}\right)\left(2 H_{k}-H_{2 k}\right) \quad \text { for each } k=1, \ldots, p-1
$$

Therefore

$$
\sum_{k=1}^{p-1} \frac{\left(\begin{array}{c}
2 k \\
k
\end{array}\right)}{k} H_{2 k}=2 \sum_{k=1}^{p-1} \frac{\left(\begin{array}{c}
2 k \\
k
\end{array}\right)}{k} H_{k}-\sum_{k=1}^{p-1} \frac{1}{k} \sum_{j=1}^{k}\left(\begin{array}{l}
k \\
j
\end{array}\right)^{2} H_{j}
$$

Observe that

$$
\sum_{k=1}^{p-1} \frac{1}{k} \sum_{j=1}^{k}\left(\begin{array}{l}
k \\
j
\end{array}\right)^{2} H_{j}=\sum_{j=1}^{p-1} \frac{H_{j}}{j} \sum_{k=j}^{p-1}\left(\begin{array}{l}
k \\
j
\end{array}\right)\left(\begin{array}{c}
k-1 \\
j-1
\end{array}\right)
$$


and

$$
\begin{aligned}
& \sum_{k=j}^{p-1}\left(\begin{array}{l}
k \\
j
\end{array}\right)\left(\begin{array}{c}
k-1 \\
j-1
\end{array}\right) \\
= & \sum_{i=0}^{p-1-j}\left(\begin{array}{c}
i+j \\
i
\end{array}\right)\left(\begin{array}{c}
i+j-1 \\
i
\end{array}\right)=\sum_{i=0}^{p-1-j}\left(\begin{array}{c}
-j-1 \\
i
\end{array}\right)\left(\begin{array}{c}
-j \\
i
\end{array}\right) \\
\equiv & \sum_{i=0}^{p-1-j}\left(\begin{array}{c}
p-j \\
i
\end{array}\right)\left(\begin{array}{c}
p-1-j \\
i
\end{array}\right)=\sum_{i=0}^{p-1-j}\left(\begin{array}{c}
p-j \\
i
\end{array}\right)\left(\begin{array}{c}
p-1-j \\
p-1-j-i
\end{array}\right) \\
= & \left(\begin{array}{c}
2 p-2 j-1 \\
p-1-j
\end{array}\right)(\bmod p)
\end{aligned}
$$

with the help of the Chu-Vandermonde identity (2.1). Thus

$$
\begin{aligned}
\sum_{k=1}^{p-1} \frac{1}{k} \sum_{j=1}^{k}\left(\begin{array}{c}
k \\
j
\end{array}\right)^{2} H_{j} & \equiv \sum_{j=1}^{p-1} \frac{H_{j}}{j}\left(\begin{array}{c}
2 p-2 j-1 \\
p-1-j
\end{array}\right) \\
& \equiv \sum_{j=1}^{p-1} \frac{H_{j}}{j}\left(\begin{array}{c}
-2 j-1 \\
p-1-j
\end{array}\right)=\sum_{j=1}^{p-1} \frac{H_{j}}{j}\left(\begin{array}{c}
p+j-1 \\
2 j
\end{array}\right)(-1)^{j} \\
& =\sum_{j=1}^{p-1} \frac{H_{j}}{j} \cdot \frac{p(-1)^{j}}{(2 j) !(p+j)} \prod_{i=1}^{j}\left(p^{2}-i^{2}\right) \\
& \equiv p \sum_{j=1}^{p-1} \frac{H_{j}}{j^{2}\left(\begin{array}{c}
2 j \\
j
\end{array}\right)} \equiv p \sum_{j=(p+1) / 2}^{p-1} \frac{H_{j}}{j^{2}\left(\begin{array}{c}
2 j \\
j
\end{array}\right)}(\bmod p) .
\end{aligned}
$$

By [S11, Lemma 2.1],

$$
j\left(\begin{array}{c}
2 j \\
j
\end{array}\right)\left(\begin{array}{c}
2(p-j) \\
p-j
\end{array}\right) \equiv 2 p\left(\bmod p^{2}\right) \quad \text { for all } j=\frac{p+1}{2}, \ldots, p-1 .
$$

(Tauraso [T] contains a similar technique.) Therefore

$$
\begin{aligned}
& \sum_{k=1}^{p-1} \frac{1}{k} \sum_{j=1}^{k}\left(\begin{array}{l}
k \\
j
\end{array}\right)^{2} H_{j} \\
\equiv & \frac{1}{2} \sum_{j=(p+1) / 2}^{p-1} \frac{H_{j}}{j}\left(\begin{array}{c}
2(p-j) \\
p-j
\end{array}\right)=\frac{1}{2} \sum_{k=1}^{(p-1) / 2} \frac{\left(\begin{array}{c}
2 k \\
k
\end{array}\right) H_{p-k}}{p-k}(\bmod p) .
\end{aligned}
$$

Since

$$
H_{p-k}=H_{p-1}-\sum_{0<j<k} \frac{1}{p-j} \equiv H_{k-1}=H_{k}-\frac{1}{k}(\bmod p)
$$


for all $k=1, \ldots, p-1$, from the above we obtain

$$
\sum_{k=1}^{p-1} \frac{1}{k} \sum_{j=1}^{k}\left(\begin{array}{l}
k \\
j
\end{array}\right)^{2} H_{j} \equiv-\frac{1}{2} \sum_{k=1}^{p-1} \frac{\left(\begin{array}{c}
2 k \\
k
\end{array}\right)}{k} H_{k}+\frac{1}{2} \sum_{k=1}^{p-1} \frac{\left(\begin{array}{c}
2 k \\
k
\end{array}\right)}{k^{2}}(\bmod p) .
$$

Combining this with (2.4) we get

$$
\sum_{k=1}^{p-1} \frac{\left(\begin{array}{c}
2 k \\
k
\end{array}\right)}{k} H_{2 k} \equiv \frac{5}{2} \sum_{k=1}^{p-1} \frac{\left(\begin{array}{c}
2 k \\
k
\end{array}\right)}{k} H_{k}-\frac{1}{2} \sum_{k=1}^{p-1} \frac{\left(\begin{array}{c}
2 k \\
k
\end{array}\right)}{k^{2}}(\bmod p) .
$$

For each $k=1, \ldots, p-1$, clearly

$$
\left(\begin{array}{l}
p \\
k
\end{array}\right)=\frac{p}{k} \prod_{0<j<k} \frac{p-j}{j} \equiv(-1)^{k-1} \frac{p}{k}\left(1-p H_{k-1}\right)\left(\bmod p^{3}\right) .
$$

Thus

$$
\sum_{k=1}^{p-1}(-1)^{k}\left(\begin{array}{l}
p \\
k
\end{array}\right)\left(\begin{array}{c}
2 k \\
k
\end{array}\right) \equiv-p \sum_{k=1}^{p-1} \frac{\left(\begin{array}{c}
2 k \\
k
\end{array}\right)}{k}\left(1-p H_{k-1}\right)\left(\bmod p^{3}\right) .
$$

On the other hand, by (2.3) we have

$$
\begin{aligned}
\sum_{k=0}^{p}(-1)^{k}\left(\begin{array}{l}
p \\
k
\end{array}\right)\left(\begin{array}{c}
2 k \\
k
\end{array}\right) & =(-1)^{p} \sum_{k=0}^{(p-1) / 2}\left(\begin{array}{c}
p \\
2 k
\end{array}\right)\left(\begin{array}{c}
2 k \\
k
\end{array}\right) \\
& \equiv-1+p \sum_{k=1}^{(p-1) / 2} \frac{1-p H_{2 k-1}}{2 k}\left(\begin{array}{c}
2 k \\
k
\end{array}\right)\left(\bmod p^{3}\right)
\end{aligned}
$$

Therefore

$$
\begin{aligned}
& -p \sum_{k=1}^{p-1} \frac{\left(\begin{array}{c}
2 k \\
k
\end{array}\right)}{k}\left(1-p H_{k}+\frac{p}{k}\right)-\left(\begin{array}{c}
2 p \\
p
\end{array}\right)+1 \\
\equiv & -1+p \sum_{k=1}^{(p-1) / 2} \frac{1-p\left(H_{2 k}-1 /(2 k)\right)}{2 k}\left(\begin{array}{c}
2 k \\
k
\end{array}\right)\left(\bmod p^{3}\right)
\end{aligned}
$$

Since $\left(\begin{array}{c}2 p \\ p\end{array}\right) \equiv 2\left(\bmod p^{3}\right)$ by Wolstenholme's theorem, and

$$
\sum_{k=1}^{p-1} \frac{\left(\begin{array}{c}
2 k \\
k
\end{array}\right)}{k} \equiv 0\left(\bmod p^{2}\right)
$$

by [ST10], from (2.6) we get

$$
\sum_{k=1}^{p-1} \frac{\left(\begin{array}{c}
2 k \\
k
\end{array}\right)}{k} H_{k} \equiv \frac{1}{2 p} \sum_{k=1}^{(p-1) / 2} \frac{\left(\begin{array}{c}
2 k \\
k
\end{array}\right)}{k}-\frac{1}{2} \sum_{k=1}^{(p-1) / 2} \frac{\left(\begin{array}{c}
2 k \\
k
\end{array}\right)}{k} H_{2 k}+\frac{5}{4} \sum_{k=1}^{(p-1) / 2} \frac{\left(\begin{array}{c}
2 k \\
k
\end{array}\right)}{k^{2}}(\bmod p) .
$$


(Note that $\sum_{k=1}^{p-1}\left(\begin{array}{c}2 k \\ k\end{array}\right) / k^{2} \equiv \sum_{k=1}^{(p-1) / 2}\left(\begin{array}{c}2 k \\ k\end{array}\right) / k^{2}(\bmod p)$.).

Clearly,

$$
p H_{2 p-2 k}=p \sum_{\substack{j=1 \\ j \neq p}}^{2 p-2 k} \frac{1}{j}+1 \equiv 1(\bmod p)
$$

for all $k=1, \ldots,(p-1) / 2$. So we have

$$
\begin{aligned}
\sum_{k=1}^{(p-1) / 2} \frac{1}{k^{2}\left(\begin{array}{c}
2 k \\
k
\end{array}\right)} & \equiv \sum_{k=1}^{(p-1) / 2} \frac{p H_{2 p-2 k}}{k^{2}\left(\begin{array}{c}
2 k \\
k
\end{array}\right)}=\sum_{j=(p+1) / 2}^{p-1} \frac{p H_{2 j}}{(p-j)^{2}\left(\begin{array}{c}
2(p-j) \\
p-j
\end{array}\right)} \\
& \equiv \frac{1}{2} \sum_{j=(p+1) / 2}^{p-1} \frac{\left(\begin{array}{c}
2 j \\
j
\end{array}\right)}{j} H_{2 j}(\bmod p)
\end{aligned}
$$

with the help of [S11, Lemma 2.1]. By [S11, (1.2) and (1.3)],

$$
\frac{1}{p} \sum_{k=1}^{(p-1) / 2} \frac{\left(\begin{array}{c}
2 k \\
k
\end{array}\right)}{k}+\sum_{k=1}^{(p-1) / 2} \frac{2}{k^{2}\left(\begin{array}{c}
2 k \\
k
\end{array}\right)} \equiv 0(\bmod p) \text {. }
$$

Therefore

$$
\sum_{k=1}^{p-1} \frac{\left(\begin{array}{c}
2 k \\
k
\end{array}\right)}{k} H_{2 k} \equiv \sum_{k=1}^{(p-1) / 2} \frac{\left(\begin{array}{c}
2 k \\
k
\end{array}\right)}{k} H_{2 k}-\frac{1}{p} \sum_{k=1}^{(p-1) / 2} \frac{\left(\begin{array}{c}
2 k \\
k
\end{array}\right)}{k}(\bmod p) .
$$

Combining this with (2.8) we get

$$
\sum_{k=1}^{p-1} \frac{\left(\begin{array}{c}
2 k \\
k
\end{array}\right)}{k} H_{k} \equiv \frac{5}{4} \sum_{k=1}^{p-1} \frac{\left(\begin{array}{c}
2 k \\
k
\end{array}\right)}{k^{2}}-\frac{1}{2} \sum_{k=1}^{p-1} \frac{\left(\begin{array}{c}
2 k \\
k
\end{array}\right)}{k} H_{2 k}(\bmod p) .
$$

(2.5) and (2.9) together imply that

$\sum_{k=1}^{p-1} \frac{\left(\begin{array}{c}2 k \\ k\end{array}\right)}{k} H_{k} \equiv \frac{2}{3} \sum_{k=1}^{p-1} \frac{\left(\begin{array}{c}2 k \\ k\end{array}\right)}{k^{2}}(\bmod p)$ and $\sum_{k=1}^{p-1} \frac{\left(\begin{array}{c}2 k \\ k\end{array}\right)}{k} H_{2 k} \equiv \frac{7}{6} \sum_{k=1}^{p-1} \frac{\left(\begin{array}{c}2 k \\ k\end{array}\right)}{k^{2}}(\bmod p)$.

It is known that

$$
\sum_{k=1}^{p-1} \frac{\left(\begin{array}{c}
2 k \\
k
\end{array}\right)}{k^{2}} \equiv \frac{1}{2}\left(\frac{p}{3}\right) B_{p-2}\left(\frac{1}{3}\right)(\bmod p)
$$

(cf. $[\mathrm{MT}]$ ). So we get the desired (1.1) and (1.2).

Remark 2.1. In [S11] the second author proved that

$$
-\frac{1}{p} \sum_{k=1}^{(p-1) / 2} \frac{\left(\begin{array}{c}
2 k \\
k
\end{array}\right)}{k} \equiv \sum_{k=1}^{(p-1) / 2} \frac{2}{k^{2}\left(\begin{array}{c}
2 k \\
k
\end{array}\right)} \equiv\left(\frac{-1}{p}\right) \frac{8}{3} E_{p-3}(\bmod p) .
$$




\section{Proof of Theorem 1.2}

Lemma 3.1. For any nonnegative integers $m$ and $n$, we have

$$
\sum_{k=0}^{n}\left(\begin{array}{c}
x+k \\
m
\end{array}\right)=\left(\begin{array}{c}
n+x+1 \\
m+1
\end{array}\right)-\left(\begin{array}{c}
x \\
m+1
\end{array}\right)
$$

and

$$
\sum_{k=0}^{n}\left(\begin{array}{l}
n \\
k
\end{array}\right)^{2}\left(\begin{array}{c}
x+k \\
2 n
\end{array}\right)=\left(\begin{array}{l}
x \\
n
\end{array}\right)^{2}
$$

Remark 3.1. Both (3.1) and (3.2) can be found in [G, (1.48) and (6.30)].

Lemma 3.2. For any nonnegative integer $n$, we have

$$
\sum_{k=0}^{n}\left(\begin{array}{l}
n \\
k
\end{array}\right)^{2}\left(\begin{array}{c}
x+k \\
2 n+1
\end{array}\right)=\frac{1}{(4 n+2)\left(\begin{array}{c}
2 n \\
n
\end{array}\right)} \sum_{k=0}^{n}(2 x-3 k)\left(\begin{array}{l}
x \\
k
\end{array}\right)^{2}\left(\begin{array}{c}
2 k \\
k
\end{array}\right) .
$$

Remark 3.2. One might wonder how we find (3.3). In fact, we use the software package Sigma to find a recurrence for $u_{n}=\sum_{k=0}^{n}\left(\begin{array}{l}n \\ k\end{array}\right)^{2}\left(\begin{array}{c}x+k \\ 2 n+1\end{array}\right)$ and then obtain (3.3) by solving the recurrence for $u_{n}$ via Sigma.

Proof of Lemma 3.2. Let $F(x)$ and $G(x)$ denote the left-hand side and the right-hand side of (3.3). With the help of (3.2), we see that

$$
F(x+1)-F(x)=\left(\begin{array}{l}
x \\
n
\end{array}\right)^{2}
$$

Applying the Zeilberger algorithm (see, e.g., [PWZ, pp. 101-119]) via Mathematica, we find that

$$
G(x+1)-G(x)=\left(\begin{array}{l}
x \\
n
\end{array}\right)^{2} \text { for all } x=0,1,2, \ldots
$$

So, by induction $F(x)=G(x)$ for all $x \in \mathbb{N}$. As $F(x)$ and $G(x)$ are polynomials in $x$ of degree $2 n+1$, we have the desired (3.3). 
Proof of Theorem 1.2. (i) With the help of Lemma 3.1, we have

$$
\begin{aligned}
\sum_{n=0}^{p-1} g_{n} & =\sum_{n=0}^{p-1} \sum_{k=0}^{n}\left(\begin{array}{l}
n \\
k
\end{array}\right)^{2}\left(\begin{array}{c}
2 k \\
k
\end{array}\right)=\sum_{k=0}^{p-1}\left(\begin{array}{c}
2 k \\
k
\end{array}\right) \sum_{n=k}^{p-1}\left(\begin{array}{l}
n \\
k
\end{array}\right)^{2} \\
& =\sum_{k=0}^{p-1}\left(\begin{array}{c}
2 k \\
k
\end{array}\right) \sum_{n=k}^{p-1} \sum_{j=0}^{k}\left(\begin{array}{c}
k \\
j
\end{array}\right)^{2}\left(\begin{array}{c}
n+j \\
2 k
\end{array}\right) \\
& =\sum_{k=0}^{p-1}\left(\begin{array}{c}
2 k \\
k
\end{array}\right) \sum_{j=0}^{k}\left(\begin{array}{c}
k \\
j
\end{array}\right)^{2} \sum_{n=k}^{p-1}\left(\begin{array}{c}
n+j \\
2 k
\end{array}\right) \\
& =\sum_{k=0}^{p-1}\left(\begin{array}{c}
2 k \\
k
\end{array}\right) \sum_{j=0}^{k}\left(\begin{array}{c}
k \\
j
\end{array}\right)^{2}\left(\begin{array}{c}
p+j \\
2 k+1
\end{array}\right) .
\end{aligned}
$$

Thus, by applying Lemma 3.2 we get

$$
\begin{aligned}
\sum_{k=0}^{p-1} g_{k} & =\sum_{k=0}^{p-1} \frac{1}{4 k+2} \sum_{j=0}^{k}(2 p-3 j)\left(\begin{array}{c}
p \\
j
\end{array}\right)^{2}\left(\begin{array}{c}
2 j \\
j
\end{array}\right) \\
& =\frac{1}{2} \sum_{j=0}^{p-1}(2 p-3 j)\left(\begin{array}{c}
p \\
j
\end{array}\right)^{2}\left(\begin{array}{c}
2 j \\
j
\end{array}\right)\left(\sum_{k=0}^{p-1} \frac{1}{2 k+1}-\sum_{0 \leqslant i<j} \frac{1}{2 i+1}\right) \\
& =\frac{1}{2} \sum_{j=0}^{p-1}(2 p-3 j)\left(\begin{array}{c}
p \\
j
\end{array}\right)^{2}\left(\begin{array}{c}
2 j \\
j
\end{array}\right)\left(H_{2 p-1}-\frac{H_{p-1}}{2}-H_{2 j}+\frac{H_{j}}{2}\right) .
\end{aligned}
$$

Note that $p H_{p-1} \equiv 0\left(\bmod p^{3}\right)$ and

$$
\begin{aligned}
p H_{2 p-1} & =1+p \sum_{j=1}^{p-1}\left(\frac{1}{p-j}+\frac{1}{p+j}\right)=1+p \sum_{j=1}^{p-1} \frac{2 p}{p^{2}-j^{2}} \\
& \equiv 1-2 p^{2} H_{p-1}^{(2)} \equiv 1\left(\bmod p^{3}\right) .
\end{aligned}
$$

Therefore

$$
\begin{aligned}
\sum_{k=0}^{p-1} g_{k} \equiv & \sum_{j=0}^{p-1} \frac{2 p-3 j}{2 p}\left(\begin{array}{c}
p \\
j
\end{array}\right)^{2}\left(\begin{array}{c}
2 j \\
j
\end{array}\right) \\
& +\sum_{j=0}^{p-1} \frac{2 p-3 j}{2}\left(\begin{array}{c}
p \\
j
\end{array}\right)^{2}\left(\begin{array}{c}
2 j \\
j
\end{array}\right)\left(\frac{H_{j}}{2}-H_{2 j}\right)\left(\bmod p^{3}\right)
\end{aligned}
$$


and hence

$$
\begin{aligned}
\sum_{k=1}^{p-1} g_{k} \equiv & \sum_{j=1}^{p-1} \frac{2 p-3 j}{2 p} \cdot \frac{p^{2}}{j^{2}}\left(\begin{array}{c}
p-1 \\
j-1
\end{array}\right)^{2}\left(\begin{array}{c}
2 j \\
j
\end{array}\right) \\
& +\sum_{j=1}^{p-1} \frac{2 p-3 j}{2} \cdot \frac{p^{2}}{j^{2}}\left(\begin{array}{c}
p-1 \\
j-1
\end{array}\right)^{2}\left(\begin{array}{c}
2 j \\
j
\end{array}\right)\left(\frac{H_{j}}{2}-H_{2 j}\right) \\
\equiv & p^{2} \sum_{j=1}^{p-1} \frac{\left(\begin{array}{c}
2 j \\
j
\end{array}\right)}{j^{2}}-\frac{3 p}{2} \sum_{j=1}^{p-1} \frac{\left(\begin{array}{c}
2 j \\
j
\end{array}\right)}{j}\left(\begin{array}{c}
p-1 \\
j-1
\end{array}\right)^{2} \\
& -\frac{3}{2} p^{2} \sum_{j=1}^{p-1} \frac{\left(\begin{array}{c}
2 j \\
j
\end{array}\right)}{j}\left(\frac{H_{j}}{2}-H_{2 j}\right)\left(\bmod p^{3}\right) .
\end{aligned}
$$

(Note that $\left(\begin{array}{c}2 j \\ j\end{array}\right) H_{2 j}$ is $p$-adic integral for all $j=1, \ldots, p-1$.) Clearly,

$$
\left(\begin{array}{c}
p-1 \\
j-1
\end{array}\right)^{2} \equiv\left(1-p H_{j-1}\right)^{2} \equiv 1-2 p H_{j-1}\left(\bmod p^{2}\right)
$$

Thus

$$
\begin{aligned}
\frac{1}{p^{2}} \sum_{k=1}^{p-1} g_{k} \equiv & \sum_{k=1}^{p-1} \frac{\left(\begin{array}{c}
2 k \\
k
\end{array}\right)}{k^{2}}-\frac{3}{2}\left(\frac{1}{p} \sum_{k=1}^{p-1} \frac{\left(\begin{array}{c}
2 k \\
k
\end{array}\right)}{k}-2 \sum_{k=1}^{p-1} \frac{\left(\begin{array}{c}
2 k \\
k
\end{array}\right)}{k}\left(H_{k}-\frac{1}{k}\right)\right) \\
& -\frac{3}{2}\left(\frac{1}{2} \sum_{k=1}^{p-1} \frac{\left(\begin{array}{c}
2 k \\
k
\end{array}\right)}{k} H_{k}-\sum_{k=1}^{p-1} \frac{\left(\begin{array}{c}
2 k \\
k
\end{array}\right)}{k} H_{2 k}\right) \\
\equiv & -2 \sum_{k=1}^{p-1} \frac{\left(\begin{array}{c}
2 k \\
k
\end{array}\right)}{k^{2}}+\frac{9}{4} \sum_{k=1}^{p-1} \frac{\left(\begin{array}{c}
2 k \\
k
\end{array}\right)}{k} H_{k}+\frac{3}{2} \sum_{k=1}^{p-1} \frac{\left(\begin{array}{c}
2 k \\
k
\end{array}\right)}{k} H_{2 k}(\bmod p)
\end{aligned}
$$

with the help of (2.7). Now, applying Theorem 1.1 and (2.10) we immediately get that

$$
\frac{1}{p^{2}} \sum_{k=1}^{p-1} g_{k} \equiv \frac{5}{8}\left(\frac{p}{3}\right) B_{p-2}\left(\frac{1}{3}\right)(\bmod p) .
$$

(ii) Observe that

$$
\begin{aligned}
\sum_{n=0}^{p-1}\left(2 g_{n}-h_{n}\right) & =\sum_{n=0}^{p-1} \sum_{k=0}^{n}\left(\begin{array}{l}
n \\
k
\end{array}\right)^{2}\left(2-\frac{1}{k+1}\right)\left(\begin{array}{c}
2 k \\
k
\end{array}\right) \\
& =\sum_{k=0}^{p-1} \frac{2 k+1}{k+1}\left(\begin{array}{c}
2 k \\
k
\end{array}\right) \sum_{n=k}^{p-1}\left(\begin{array}{l}
n \\
k
\end{array}\right)^{2} .
\end{aligned}
$$


Similar to the proof in part (i), we have

$$
\begin{aligned}
\sum_{n=0}^{p-1}\left(2 g_{n}-h_{n}\right) & =\sum_{k=0}^{p-1} \frac{(2 k+1) /(k+1)}{4 k+2} \sum_{j=0}^{k}(2 p-3 j)\left(\begin{array}{c}
p \\
j
\end{array}\right)^{2}\left(\begin{array}{c}
2 j \\
j
\end{array}\right) \\
& =\frac{1}{2} \sum_{j=0}^{p-1}(2 p-3 j)\left(\begin{array}{c}
p \\
j
\end{array}\right)^{2}\left(\begin{array}{c}
2 j \\
j
\end{array}\right)\left(H_{p-1}+\frac{1}{p}-H_{j}\right)
\end{aligned}
$$

and thus

$$
\begin{aligned}
& \sum_{k=1}^{p-1}\left(2 g_{k}-h_{k}\right) \\
\equiv & \frac{1}{2} \sum_{j=1}^{p-1}(2 p-3 j) \frac{p^{2}}{j^{2}}\left(\begin{array}{c}
p-1 \\
j-1
\end{array}\right)^{2}\left(\begin{array}{c}
2 j \\
j
\end{array}\right)\left(\frac{1}{p}-H_{j}\right) \\
\equiv & p^{2} \sum_{j=1}^{p-1} \frac{\left(\begin{array}{c}
2 j \\
j
\end{array}\right)}{j^{2}}-\frac{3 p}{2} \sum_{j=1}^{p-1} \frac{\left(\begin{array}{c}
2 j \\
j
\end{array}\right)}{j}\left(1-2 p H_{j-1}\right)\left(1-p H_{j}\right)(\text { by }(3.4)) \\
\equiv & p^{2} \sum_{j=1}^{p-1} \frac{\left(\begin{array}{c}
2 j \\
j
\end{array}\right)}{j^{2}}-\frac{3}{2} p \sum_{j=1}^{p-1} \frac{\left(\begin{array}{c}
2 j \\
j
\end{array}\right)}{j}\left(1+p\left(\frac{2}{j}-3 H_{j}\right)\right) \\
= & -2 p^{2} \sum_{j=1}^{p-1} \frac{\left(\begin{array}{c}
2 j \\
j
\end{array}\right)}{j^{2}}-\frac{3}{2} p \sum_{j=1}^{p-1} \frac{\left(\begin{array}{c}
2 j \\
j
\end{array}\right)}{j}+\frac{9}{2} p^{2} \sum_{j=1}^{p-1} \frac{\left(\begin{array}{c}
2 j \\
j
\end{array}\right)}{j} H_{j}\left(\bmod p^{3}\right)
\end{aligned}
$$

Combining this with (1.1), (2.7) and (2.10), we obtain that

$$
\sum_{k=1}^{p-1}\left(2 g_{k}-h_{k}\right) \equiv \frac{p^{2}}{2}\left(\frac{p}{3}\right) B_{p-2}\left(\frac{1}{3}\right)\left(\bmod p^{3}\right) .
$$

Thus,

$$
\sum_{k=1}^{p-1} h_{k} \equiv \frac{3}{4} p^{2}\left(\frac{p}{3}\right) B_{p-2}\left(\frac{1}{3}\right)\left(\bmod p^{3}\right)
$$

with the help of (3.5).

(iii) By [S14b, Theorem1.1],

$$
\sum_{k=1}^{p-1} g_{k} \equiv p^{2} \sum_{k=1}^{p-1} g_{k} H_{k}^{(2)}+\frac{7}{6} p^{3} B_{p-3}\left(\bmod p^{4}\right) .
$$

Therefore

$$
\sum_{k=1}^{p-1} g_{k} H_{k}^{(2)} \equiv \frac{1}{p^{2}} \sum_{k=1}^{p-1} g_{k}(\bmod p)
$$

and hence (1.6) holds in view of (3.5). 
From [S14b, Theorem1.1] we know that

$$
\sum_{k=0}^{p-1} g_{k}(x)\left(1-p^{2} H_{k}^{(2)}\right) \equiv \sum_{k=0}^{p-1} \frac{p}{2 k+1}\left(1-2 p^{2} H_{k}^{(2)}\right) x^{k}\left(\bmod p^{4}\right),
$$

where $g_{k}(x)=\sum_{j=0}^{k}\left(\begin{array}{c}k \\ j\end{array}\right)^{2}\left(\begin{array}{c}2 j \\ j\end{array}\right) x^{j}$. Therefore, the left-hand side of (3.8) minus the right-hand side of (3.8) can be written as $p^{4} P(x)$, where $P(x)$ is a polynomial of degree at most $p-1$ with $p$-adic integer coefficients. Since

$$
h_{n}=\sum_{k=0}^{n}\left(\begin{array}{l}
n \\
k
\end{array}\right)^{2}\left(\begin{array}{c}
2 k \\
k
\end{array}\right) C_{k}=\int_{0}^{1} g_{n}(x) d x \text { for } n=0,1,2, \ldots,
$$

we deduce that

$$
\begin{aligned}
& \sum_{k=0}^{p-1} h_{k}\left(1-p^{2} H_{k}^{(2)}\right) \\
= & \int_{0}^{1} \sum_{k=0}^{p-1} g_{k}(x)\left(1-p^{2} H_{k}^{(2)}\right) d x \\
= & \sum_{k=0}^{p-1} \frac{p}{2 k+1}\left(1-2 p^{2} H_{k}^{(2)}\right) \int_{0}^{1} x^{k} d x+p^{4} \int_{0}^{1} P(x) d x \\
\equiv & \sum_{k=0}^{p-1} \frac{2 p}{2 k+1}\left(1-2 p^{2} H_{k}^{(2)}\right)-\sum_{k=0}^{p-1} \frac{p}{k+1}\left(1-2 p^{2} H_{k}^{(2)}\right)\left(\bmod p^{3}\right) .
\end{aligned}
$$

Combining (3.7) and (3.8) we see that

$$
\begin{aligned}
1+\frac{7}{6} p^{3} B_{p-3} & \equiv \sum_{k=0}^{p-1} g_{k}\left(1-p^{2} H_{k}^{(2)}\right) \\
& \equiv \sum_{k=0}^{p-1} \frac{p}{2 k+1}\left(1-2 p^{2} H_{k}^{(2)}\right)\left(\bmod p^{4}\right) .
\end{aligned}
$$

Therefore

$$
\begin{aligned}
\sum_{k=0}^{p-1} h_{k}\left(1-p^{2} H_{k}^{(2)}\right) & \equiv 2+\frac{7}{3} p^{3} B_{p-3}-\sum_{k=0}^{p-1} \frac{p}{k+1}+2 p^{3} \sum_{k=0}^{p-1} \frac{H_{k}^{(2)}}{k+1} \\
& \equiv 2-1-p H_{p-1}+2 p^{2} H_{p-1}^{(2)} \equiv 1\left(\bmod p^{3}\right)
\end{aligned}
$$

which implies that

$$
\sum_{k=1}^{p-1} h_{k}\left(1-p^{2} H_{k}^{(2)}\right) \equiv 0\left(\bmod p^{3}\right)
$$


Combining this (3.6) we obtain the desired (1.7).

So far we have completed the proof of Theorem 1.2.

Acknowledgment. The authors would like to thank the referee for helpful comments.

\section{REFERENCES}

[B] P. Barrucand, A combinatorial identity, problem 75-4, SIAM Review 17 (1975), 168.

[G] H. W. Gould, Combinatorial Identities, Morgantown Printing and Binding Co., 1972.

[MT] S. Mattarei and R. Tautaso, Congruences for central binomial sums and finite polylogarithms, J. Number Theory 133 (2013), 131-157.

[PWZ] M. Petkovšek, H. S. Wilf and D. Zeilberger, $A=B$, A K Peters, Wellesley, 1996.

[S11] Z.-W. Sun, Super congruences and Euler numbers, Sci. China Math. 54 (2011), 2509-2535.

[S12a] Z.-W. Sun, Arithmetic theory of harmnic numbers, Proc. Amer. Math. Soc. 140 (2012), 415-428.

[S12b] Z.-W. Sun, On sums of Apéry polynomials and related congruences, J. Number Theory 132 (2012), 2673-2699.

[S12c] Z.-W. Sun, On sums of binomial coefficients modulo $p^{2}$, Colloq. Math. 127 (2012), 39-54.

[S13] Z.-W. Sun, Connections between $p=x^{2}+3 y^{2}$ and Franel numbers, J. Number Theory 133 (2013), 2914-2928.

[S14a] Z.-W. Sun, New series for some special values of $L$-functions, preprint, arXiv: $1010.4298 \mathrm{v} 6,2014$.

[S14b] Z.-W. Sun, Congruences involving $g_{n}(x)=\sum_{k=0}^{n}\left(\begin{array}{c}n \\ k\end{array}\right)^{2}\left(\begin{array}{c}2 k \\ k\end{array}\right) x^{k}$, Ramanujan J., in press. Doi:10.1007/s11139-015-9727-3

[ST10] Z.-W. Sun and R. Tauraso, New congruences for central binomial coefficients, Adv. in Appl. Math. 45 (2010), 125-148.

[ST11] Z.-W. Sun and R. Tauraso, On some new congruences for binomial coefficients, Int. J. Number Theory 7 (2011), 645-662.

[T] R. Tauraso, More congruences for central binomial coefficients, J. Number Theory 130 (2010), 2639-2649.

[W] J. Wolstenwholme, On certain properties of prime numbers, Quart. J. Appl. Math 5 (1862), 35-39.

[Z] D. Zagier, Integral solutions of Apéry-like recurrence equations, in: Groups and Symmetries: from Neolithic Scots to John McKay, CRM Proc. Lecture Notes 47, Amer. Math. Soc., Providence, RI, 2009, pp. 349-366.

(Guo-Shuai Mao) Department of Mathematics, Nanjing University, Nanjing 210093, People's Republic of China

E-mail address: mg1421007@smail.nju.edu.cn

(Zhi-Wei Sun) Department of Mathematics, Nanjing University, NanJing 210093, PeOple's Republic of China

E-mail address: zwsun@nju.edu.cn 